
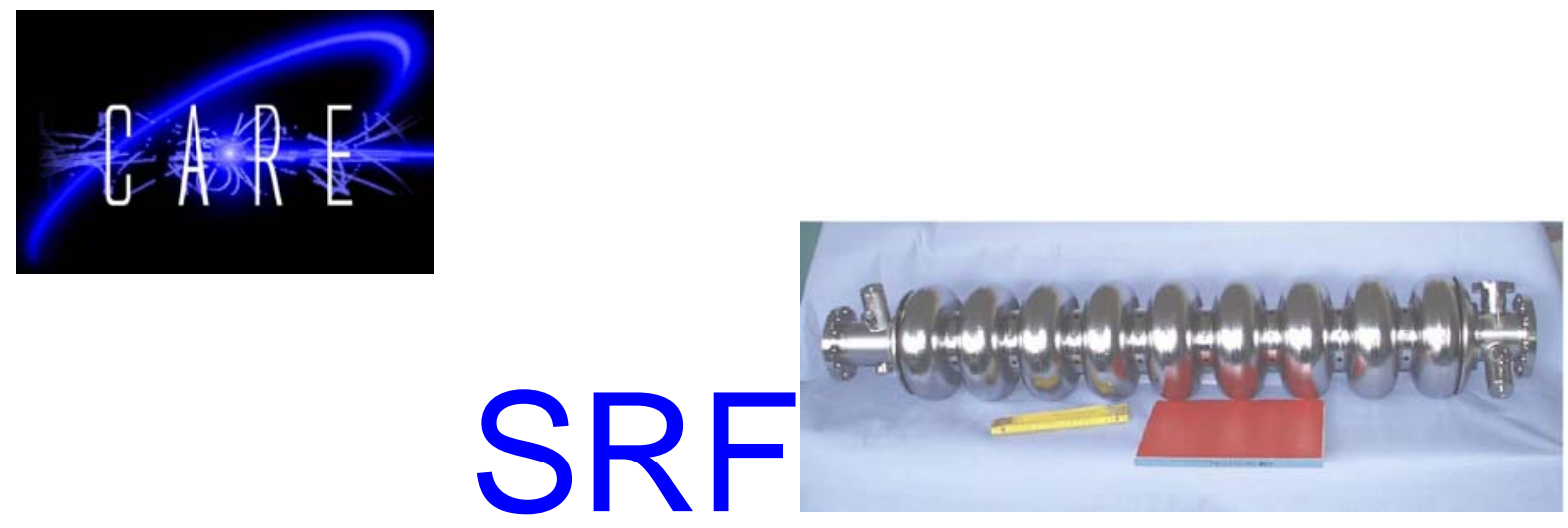

\title{
Progress in Use of Ultra-High Vacuum Cathodic Arcs for Deposition of Thin Film Superconducting Layers
}

\author{
J. Langner ${ }^{1}$, M.J. Sadowski ${ }^{1}$, P. Strzyzewski ${ }^{1}$, R. Mirowski ${ }^{1}$, J. Witkowski ${ }^{1}$, \\ S. Tazzari ${ }^{2}$, L. Catani ${ }^{2}$, A. Cianchi ${ }^{2}$, J. Lorkiewicz ${ }^{2}$, R. Russo ${ }^{3}$, \\ T. Paryjczak ${ }^{4}$, J. Rogowski $^{4}$ and J. Sekutowicz ${ }^{5}$
}

1) The Andrzej Soltan Institute for Nuclear Studies, 05-400 Otwock-Swierk, Poland.

2) University Tor Vergata and INFN Roma 2, 00-133 Rome, Italy.

3) University of Napoli and INFN-NA, Napoli, Italy.

4) Technical University of Lodz, Zeromskiego 116, 90-924 Lodz, Poland

5) DESY-MHF, 22-603 Hamburg, Germany.

\begin{abstract}
The paper reports on recent progress in the application of the UHV arc technology, which was proposed as an alternative solution for the deposition of thin superconducting films of pure niobium $(\mathrm{Nb})$ upon the inner surfaces of RF cavities designed for particle accelerators. There are presented new experimental studies aimed at the deposition of superconducting films of pure niobium $(\mathrm{Nb})$ and lead $(\mathrm{Pb})$ needed for the modern accelerator technology. The main experimental results and characteristics of arc-deposited thin superconducting films are discussed, and the progress achieved recently in the formation of such films is presented.
\end{abstract}

\section{Contribution to the XXII ${ }^{\text {th }}$ "International Symposium on \\ Discharges and Electrical Insulation in Vacuum" \\ Matsue (Japan), 25-29 September 2006}

Work supported by the European Community-Research Infrastructure Activity under the FP6 "Structuring the European Research Area" programme (CARE, contract number RII3-CT2003-506395) 


\title{
Progress in Use of Ultra-High Vacuum Cathodic Arcs for Deposition of Thin Film Superconducting Layers
}

\author{
J. Langner ${ }^{1)}$, M.J. Sadowski ${ }^{1)}$, P. Strzyzewski ${ }^{1)}$, R. Mirowski ${ }^{1)}$, J. Witkowski ${ }^{1)}$, \\ S. Tazzari $^{2)}$, L. Catani $^{2}$, A. Cianchi ${ }^{2}$, J. Lorkiewicz ${ }^{2)}$, R. Russo ${ }^{3)}$, \\ T. Paryjczak ${ }^{4)}$ J. Rogowski ${ }^{4)}$ and J. Sekutowicz ${ }^{5)}$ \\ 1) The Andrzej Soltan Institute for Nuclear Studies, 05-400 Otwock-Swierk, Poland. \\ ${ }^{2)}$ University Tor Vergata and INFN Roma 2, 00-133 Rome, Italy. \\ 3) University of Napoli and INFN-NA, Napoli, Italy. \\ 4) Technical University of Lodz, Zeromskiego 116, 90-924 Lodz, Poland \\ 5) DESY-MHF, 22-603 Hamburg, Germany.
}

\begin{abstract}
The paper reports on recent progress in the application of the UHV arc technology, which was proposed as an alternative solution for the deposition of thin superconducting films of pure niobium $(\mathrm{Nb})$ upon the inner surfaces of RF cavities designed for particle accelerators. There are presented new experimental studies aimed at the deposition of superconducting films of pure niobium $(\mathrm{Nb})$ and lead $(\mathrm{Pb})$ needed for the modern accelerator technology. The main experimental results and characteristics of arc-deposited thin superconducting films are discussed, and the progress achieved recently in the formation of such films is presented.
\end{abstract}

\section{INTRODUCTION}

Vacuum arc technology is widely used in scientific laboratories and industry in order to produce different coatings on various surfaces. The deposition of pure and clean metal films of different types as well as possibility of the vacuum arc operation within a reactive-gas environment (in order to form compound films, such as nitrides, oxides and carbonaceous layers), make this technology very attractive. Appropriate surface layers can be formed on constructional parts of complicated shapes, ensuring high bonding strength and corrosion resistance. In a comparison with other deposition techniques, e.g. the known Physical Vapor Deposition (PVD) processes when ions have energy of a few $\mathrm{eV}$, vacuum arc discharges can produce ions of higher kinetic energies ranging from about $15 \mathrm{eV}$ to about 150 $\mathrm{eV}$ [1]. It results in the formation of a denser film and it strongly reduces surface defects, such as voids and columnar growth. The main drawback is represented by the production of micro-droplets in the near-cathode space, which become embedded in the film and increase its surface roughness. In order to eliminate the microdroplets from vacuum-arc plasmas, one can apply different magnetic filters. The main idea is to separate the plasma stream by means of an appropriate magnetic field and to eliminate micro-droplets, which (due to their large masses) move from the cathode along almost straight lines.

In connection with $R \& D$ programs concerning the construction of large linear accelerators, particular attention was paid to possibilities of the deposition of thin super-conducting layers, e.g. by the magnetron sputtering [2]. The adhesion of such layers to surfaces of the accelerator resonance cavities appeared to be not very strong. Therefore, a new concept of the deposition of thin super-conducting layers by arc discharges under Ultra-High Vacuum (UHV) conditions was proposed several years ago [3-4]. A considerable progress in the development of this technology has been achieved recently, including a problem of an effective elimination of micro-droplets in planar-arc facilities. Our previous report [5] describes concepts of 2 types of the magnetic filters suited for UHV conditions. Some experimental research on them is still under realization, in order to improve efficiency of micro-droplets filtering and plasma transport through the magnetic channel. In fact the filters with the water cooling system work very stably and their constructions enable a long-lasting operation. Additionally, it is possible to bake out the inner surface of the filter when clean conditions are required.

Another paper [6] has been devoted to residual gas measurements in a vacuum chamber at the UHV conditions: before, during and just after vacuum arc deposition process. Moreover, a comparison of gas compositions between $\mathrm{HV}$ and UHV has been presented. Such an analysis is very important when very clean layers of getter metals are needed (e.g. superconducting niobium films). Filtered vacuum arc technology in UHV opens a new road to many applications when very pure metallic and/or superconducting films are needed. 
This paper reports on recent progress in the application of the UHV arc technology, which was proposed as an alternative solution for the deposition of thin superconducting films of pure niobium $(\mathrm{Nb})$ upon the inner surfaces of RF cavities designed for particle accelerators. The status of our joint research on the deposition of such superconducting films, designed for RF accelerator cavities of the TESLA type, is described. There are also presented new experimental studies aimed at the deposition of superconducting films of pure lead $(\mathrm{Pb})$ for the formation of photo-cathodes needed for modern accelerator injectors. The main results and characteristics of arc-deposited thin superconducting films are discussed, and the progress achieved recently in the formation of such films is presented.

\section{EXPERIMENTAL SET-UP}

A typical plasma arc source with a planar cathode (shown in Fig.1) consists of a "planar" (in fact a truncated cone) cathode fixed upon a water-cooled support placed inside a vacuum chamber. To reduce an amount of micro-droplets the use is often made of a special magnetic filter, which collects the microdroplets and deflects a plasma-ion stream. Such planar arc sources were constructed both at the Tor Vergata laboratory in Rome and at the IPJ in Swierk. The detailed description of their constructions and performance can be found in our previous papers [3-7].

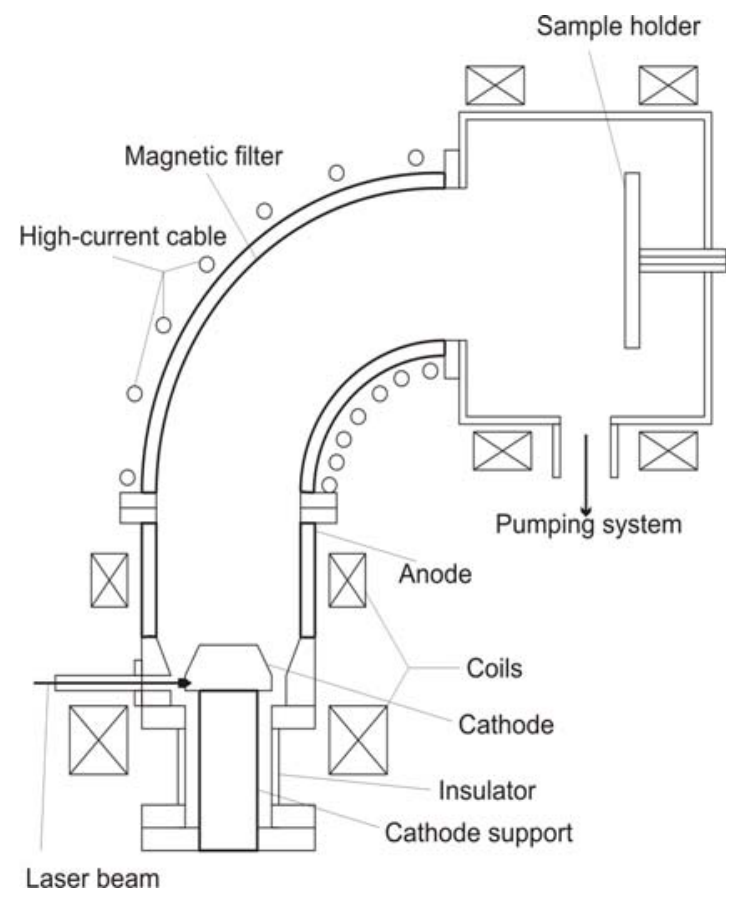

Fig. 1. Scheme of a UHV arc facility with a planar cathode and a knee-type magnetic filter.

The described facility was equipped with an oil-free pumping system consisting of a two-stage fore-vacuum pump and a turbo-molecular pump. The achievement of very good vacuum has been of primary importance, because the previous studies showed that thin niobium films, as deposited by arc discharges under UHV conditions, have properties comparable with the pure bulk niobium [7]. In our UHV facility the basic pressure $<10^{-11} \mathrm{hPa}$ was achieved after 24 hours of baking at $150^{\circ} \mathrm{C}$. In order to improve the operation of the described UHV facility we considered and tested different magnetic filters. Dimensions of the filters were chosen according to the constructional parameters of the exploited planar arc sources and according to UHV flange- and sealing-standards. For the arc ignition one must produce a small plasma burst of a sufficient density in order to form a high-conductivity path between cathode and anode, which in our case is generated by a $50-\mathrm{mJ} \mathrm{Nd}-\mathrm{YAG}$ pulsed laser focused upon the cathode surface. Such a triggering system provides the ultra-clean and reliable ignition.

\section{FORMATION AND PROPERTIES OF UHV ARC-DEPOSITED SUPERCONDUCTING THIN FILM}

In our filtered vacuum-arc systems there can be coated up to four sapphire- and $\mathrm{Cu}$-substrates simultaneously. The samples can be mounted upon a sample holder consisting of a massive $\mathrm{Cu}$ (OFHC) flange (see Fig.1) and kept at a constant temperature during the whole deposition process. The sample holder is electrically insulated from walls of the vacuum chamber, so that a bias of 20-100 V, both in DC and $\mathrm{kHz}$ pulse regime, can be applied to the coated substrates. The lowest possible arc current for the stable operation in the applied DC mode has been found to be about 60 A for niobium and only 23 A for lead, while the available cooling system of the anode has an upper limit of the arc current equal to about 140 A. The deposition rate achievable with the system operated with arc currents of $80-100 \mathrm{~A}$ is about $1 \mathrm{~nm} / \mathrm{s}$. The sample temperature during depositions is usually recorded by means of thermocouples. Most samples have been deposited at temperatures close to the room temperature, and only a few samples have been investigated at higher temperatures $\left(100-200{ }^{\circ} \mathrm{C}\right)$. As above mentioned, the residual pressure in our systems is usually set within the $10^{-11} \mathrm{hPa}$ range. The pressure usually increases to $10^{-6}-$ $10^{-7} \mathrm{hPa}$ when the arc discharge starts and it remains almost stable at the latter value throughout the deposition process. The gas pressure rise during the arc discharge is found to be almost exclusively caused by hydrogen, which partial pressure is usually more than 3 orders of magnitude higher than that of other contaminants [7].

The UHV arc-deposited Nb-layers upon sapphire substrates have been characterized by measuring their critical temperature $T_{c}$ and Residual Resistivity Ratio (RRR, defined as the ratio of the resistivity at a room temperature to that measured at $10 \mathrm{~K}$ ). Both parameters are in general very sensitive to impurities, e.g. very small amounts of oxygen in the $\mathrm{Nb}$-film can lower its $\mathrm{T}_{\mathrm{c}}$ value significantly. The RRR values of our $1.5 \mu \mathrm{m}$-thick $\mathrm{Nb}$ films, which were deposited upon the sapphire substrates at a room temperature, under the typical UHV conditions described above, range from 20 up to 50. A record value of $\mathrm{RRR}=80$ was obtained by heating up the substrate to the temperature of $150^{\circ} \mathrm{C}$ [8]. The critical temperature $\left(T_{c}\right)$ and critical current density $\left(\mathrm{J}_{\mathrm{c}}\right)$ 
values of the deposited $\mathrm{Nb}$ films were measured by means of an inductive method. The best samples have shown the values identical to bulk $\mathrm{Nb}$, i.e. $\mathrm{T}_{\mathrm{c}}=9.26 \mathrm{~K}$, $\Delta \mathrm{T}_{\mathrm{c}}=0.02 \mathrm{~K}$ and $\mathrm{J}_{\mathrm{c}}=3 \times 10^{7} \mathrm{~A} / \mathrm{cm}^{2}[10]$.

Scanning Electron Microscopy (SEM) is a very useful tool to perform the surface quality inspection for small-scale defects and to look at the surface structure. Figures $2 \mathrm{a}$ and $2 \mathrm{~b}$ show the SEM pictures of niobium layers, which were taken with different magnifications. One can see that surface presented on the Fig. 2a is very homogeneous and dense. A lack of micro-droplets upon the surface is the confirmation of good plasma filtering. Using the higher magnification (Fig. 2b) it is possible to analyze the surface structure. One can see the longitudinal shape of surface grains. The roughness of the arc deposited $\mathrm{Nb}$ samples on sapphire was found to be of the order of few tenth of a nm
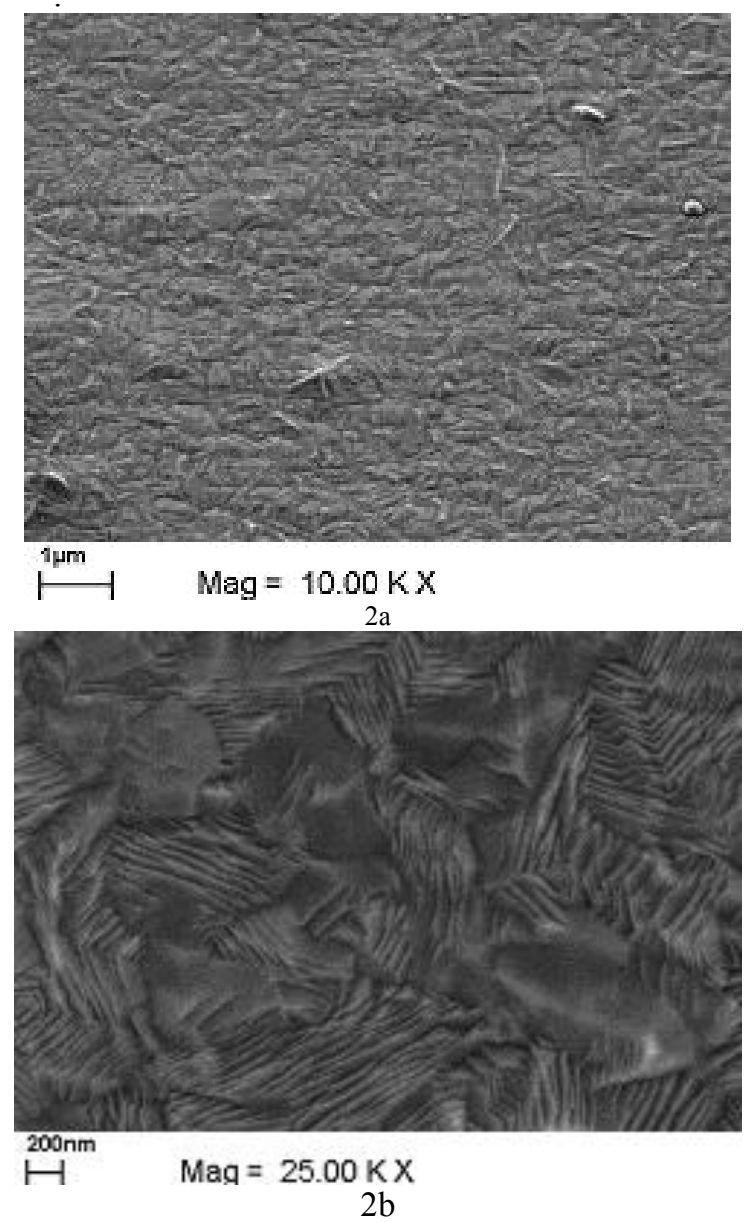

Fig. 2. SEM pictures of the surface of a niobium film, which was arcdeposited upon the sapphire substrate.

Information about the surface chemical composition and depth profile were obtained by means of a time-offlight (ToF) SIMS mass spectrometer, delivered by the Ion-ToF GmbH, Muenster, Germany. The instrument was equipped with an $\mathrm{O}_{2}+$ primary-ion gun and the high -resolution ToF mass analyzer. During the performed analysis the sample surface was irradiated with pulses of $1 \mathrm{keV}$ ions, and an average ion current amounted to 40 nA. Secondary ions emitted from the bombarded surface were mass-separated and counted with the ToF analyzer. Some results of the measurements of $\mathrm{Nb}$ - and $\mathrm{Pb}$-films are presented in Figs $3 \mathrm{a}$ and $3 \mathrm{~b}$, respectively. One can easily see that the deposited Nb-layers were clean enough. Chemical compositions of both samples appeared to be similar ones, and the deposited layers consisted mainly of $\mathrm{Nb}, \mathrm{NbO}, \mathrm{NbH}$ and $\mathrm{NbOH}$ species. The presence of some heavy impurities (like Cs, $\mathrm{Na}$ and $\mathrm{K}$ species) has also been confirmed, but their amounts were very low (not shown in Fig. 3). Typical for $\mathrm{Nb}$ layer is a relatively high level of the oxidation in the near-surface layer (see Fig. 3a). This phenomenon is not visible upon the $\mathrm{Pb}$ layer (Fig. 3b).

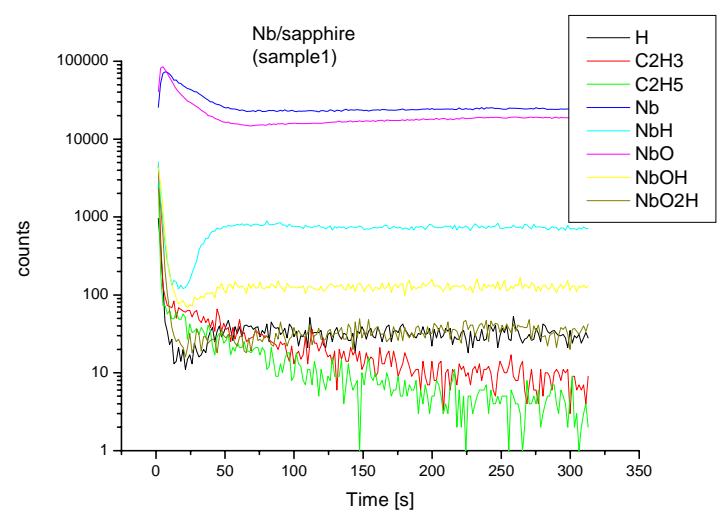

$3 a$

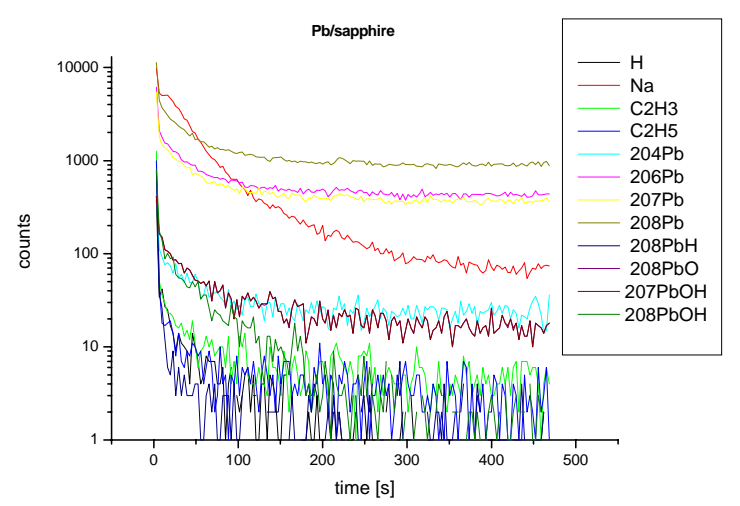

$3 b$

Fig. 3. Result of SIMS measurement of $\mathrm{Nb}(3 \mathrm{a})$ and $\mathrm{Pb}(3 \mathrm{~b})$ layers.

An alternative method to determine the purity of our films appeared to be a Glow Discharge - Optical Emission Spectroscopy (GD-OES) technique. It is generally used for the quantitative determination of surface coatings, hidden interfaces, layers and depth profiles. For the measurements to be described below the use was made of a JOBIN-YVON 8000RF device. Unfortunately, due to a lack of an appropriate $\mathrm{Nb}$-lines detector, only the deposited $\mathrm{Pb}$-layer could be analyzed by means of the GD-OES technique. The result has been shown in Fig. 4.

Due to rather small dimensions of the applied sapphire samples, which did not fit to requirements of the GD-OES device, it was necessary to use a substrate made of oxygen free copper (OFHC $\mathrm{Cu}$ ). It was coated together the sapphire substrate during the same process. 


\section{REFERENCES}

Therefore, a comparison of GD-OES results with those obtained by means of SIMS (presented in Fig. 3b) can be performed. The described results have also confirmed the high cleanliness of the $\mathrm{Pb}$ superconducting film deposited by means of the UHV arc technology.

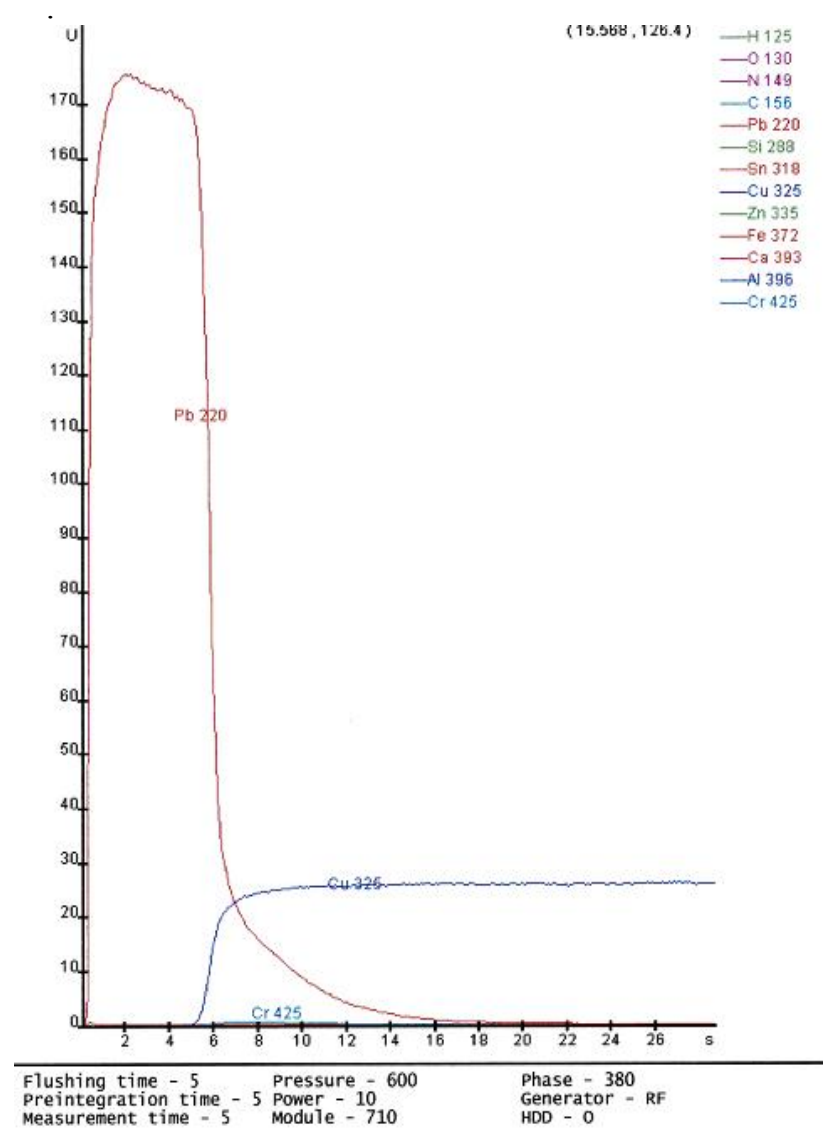

Fig. 4. GD-OES analysis of the $\mathrm{Pb}$ layer profile, which was deposited upon the $\mathrm{Cu}$-substrate under the same conditions as the sapphire ones.

\section{SUMMARY}

On the basis of the experimental studies described above, it can be concluded that the deposition processes, which are realized by means of arc discharges performed under the UHV conditions, can guarantee a very low level of unwanted impurities within the deposited films.

This statement has been confirmed by very good superconducting properties and results of the purity measurements, as described in this paper. Therefore, it opens a new road to many applications where dense, high-quality and very pure metallic-films are needed, e.g. in micro-electronics, nanotechnology, medicine etc.

\section{ACKNOWLEDGEMENT}

We acknowledge the support of the European Community-Research Infrastructure Activity under the FP6 "Structuring the European Research Area" program (CARE, contract number RII3-CT-2003-506395).

We also wish to acknowledge the assistance of Dr. E. Fortuna from the Warsaw Technical University, Poland, in the collection of the SEM pictures.
[1] S. Anders et al., Surf. Coat. Tech. 156 (2002) 3.

[2] C. Benvenuti "Superconducting coating for accelerating RF cavities: past present and future", Particle Accelerators Vol. 40 (1992), p. 43.

[3] J. Langner, M.J. Sadowski, K. Czaus, R. Mirowski, J. Witkowski, L. Catani, A. Cianchi, R. Russo, S. Tazzari, D. Proch, N.N. Koval, „Research on initiation of ultra-high vacuum arc discharges applied for deposition of thin superconducting layers", Proc. International Conference on Plasma Research and Applications PLASMA-2003, Warsaw, Poland, September 9-12, 2003.

[4] J. Langner, M.J. Sadowski, K. Czaus, R. Mirowski, J. Witkowski, L. Catani, A. Cianchi, R. Russo, F. Tazzioli, S. Tazzari, D. Proch, N.N. Koval, Y.H. Akhmadeev "Super-conducting niobium films produced by means of uhv arc", Czech. J. Phys. 54, Suppl. C (2004) C914.

[5] P. Strzyżewski, J. Langner, R. Mirowski, M.J. Sadowski, S. Tazzari and J. Witkowski, ,Magnetic Filters in UHV ArcDischarges: Constructions, Field Modelling and Tests of Efficie ncy", Physica Scripta T123 (2006) 135-139.

[6] P. Strzyzewski, L. Catani, A. Cianchi, J. Langner, J. Lorkiewicz, R. Mirowski, R. Russo, M. Sadowski, S. Tazzari and J. Witkowski, "Behaviour Of Gas Conditions During Vacuum Arc Discharges Used For Deposition Of Thin Films", AIP CP 812 (2006) 485-488.

[10] R. Russo, L. Catani, A. Cianchi, S. Tazzari and J. Langner; "High quality superconducting niobium films produced by an ultra high vacuum cathodic arc", Supercond. Sci. Technol. 18 (2005) L41-L44.

\section{E-mails of corresponding authors:}

langner@ipj.gov.pl \& msadowski@ipj.gov.pl 